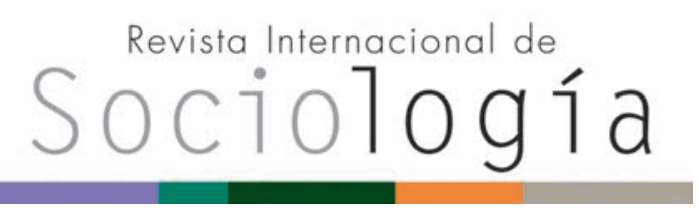

Revista Internacional de Sociología RIS

vol. 75 (3), e069, julio-septiembre, 2017, ISSN-L:0034-9712

doi: http://dx.doi.org/10.3989/ris.2017.75.3.16.61

\section{EL DECLIVE DE LA FE EN EL PROGRESO. \\ Posmaterialismo, ideología y religiosidad en las representaciones sociales de la tecnociencia}

\author{
Cristóbal Torres Albero \\ Universidad Autónoma Madrid, España. \\ cristobal.torres@uam.es \\ ORCID iD: http://orcid.org/0000-0001-5630-9101
}

Josep A. Lobera

Universidad Autónoma Madrid, España.

josep.lobera@uam.es

ORCID iD: http://orcid.org/0000-0002-0620-6312

Cómo citar este artículo / Citation: Torres Albero, C. y J. A. Lobera. 2017. "El declive de la fe en el progreso. Posmaterialismo, ideología y religiosidad en las representaciones sociales de la tecnociencia". Revista Internacional de Sociología 75 (3): e069. doi: http://dx.doi.org/10.3989/ ris.2017.75.3.16.61

\begin{abstract}
Resumen
En las sociedades contemporáneas, las representaciones sociales de la tecnociencia han devenido más complejas y poliédricas, alejándose de las concepciones meliorativas tradicionales. Nuestro trabajo profundiza en el análisis de la creciente importancia de la ambivalencia, así como en el cuestionamiento del modelo del déficit cognitivo como teoría explicativa. Apuntamos que los esquemas cognitivos vinculados a valores posmaterialistas y al nivel de religiosidad tienen un papel decisivo en la conformación de los posicionamientos de la opinión pública sobre aspectos controvertidos de la tecnociencia. Por otro lado, constatamos que estos vínculos varían según las distintas culturas nacionales. En el caso de España, la problematización de las representaciones sociales de la tecnociencia suele estar asociada, de un lado, a las personas con menores niveles de religiosidad y, de otro, con un aumento de las preocupaciones posmaterialistas debido al proceso de secularización de nuestra sociedad.
\end{abstract}

\section{Palabras Clave}

Representaciones sociales; Sociedad de la información; Tecnociencia; Valores posmaterialistas.

\section{THE DECLINE OF FAITH IN PROGRESS. \\ Posmaterialism, ideology and religiosity in the social representations of technoscience}

Copyright: @ 2017 CSIC. Este es un artículo de acceso abierto distribuido bajo los términos de la licencia Creative Commons Attribution (CC BY) España 3.0.

Recibido: 14/03/2015. Aceptado: 15/02/2017

\section{KEYWORDS}

Informational Society; Post-Materialist Values; Social Representations; Technoscience. 


\section{INTRODUCCIÓN}

La tecnociencia ha sido uno de los aspectos centrales, si no el fundamental, en los procesos de cambio social contemporáneos. Su centralidad se materializa, por un lado, en su presencia cada más extensa y cotidiana, incluso incorporándose a los cuerpos de muchos individuos a través de prótesis, sensores o marcapasos; por el otro, en el hecho de que los valores tecnocientíficos hayan permeado en todos los ámbitos de la sociedad a través de la racionalización y tecnificación de las profesiones, llegando a ser hoy estos valores tecnocientíficos una fuerza aglutinante y de cohesión social en el creciente proceso centrífugo de especialización en la sociedad. Durante siglos, el profundo impacto de la tecnociencia ha trasformado la representación social del mundo (Weltanschauung), habitando un mundo desencantado (Weber) y generalizando la creencia en un progreso prácticamente ilimitado que surge de la aplicación de la tecnociencia sobre la naturaleza -concebido por Francis Bacon en su Nueva Atlántida, donde una comunidad científica, la Casa de Salomón, dirige la sociedad redimida-.

Sin embargo, a lo largo del último medio siglo, se ha señalado la erosión de la legitimidad de la ciencia como forma de conocimiento dominante en las sociedades contemporáneas desde diferentes perspectivas (Lyotard 1984; Beck 1986). Las representaciones sociales de la tecnociencia ya no se caracterizan por una fe incondicional en sus bondades, sino por la preeminencia de una cierta ambivalencia en la mayoría de las sociedades avanzadas. En España, tan solo el $25 \%$ de la población está totalmente de acuerdo con que los beneficios de la ciencia y la tecnología son mayores que sus efectos perjudiciales, dentro de una escala de cinco posiciones; el resto matiza, en mayor o menor medida, el equilibrio general entre los aspectos positivos y negativos de la ciencia y la tecnología (Eurobarómetro 2005). La opinión pública, así, no arroja una visión monolítica y aproblemática de la tecnociencia, como se derivaba de su concepción ilustrada, por lo que el análisis de la ambivalencia en las representaciones sociales de la tecnociencia resulta ineludible (Torres-Albero 2005a y 2005b; Kerschner y Ehlers 2016).

El interés por entender las posiciones críticas hacia la tecnociencia se ha plasmado en diferentes aproximaciones teóricas. La tradicional teoría del déficit cognitivo ha sido la explicación predominante y objeto de un prolífico debate (Sturgis y Allum 2004). Nuestra investigación pretende profundizar en este debate, aportando pruebas que muestran la importancia de aspectos ideológicos y axiológicos en la explicación de las posiciones críticas hacia la tecnociencia, más allá del nivel conocimiento tecnocientífico de los individuos. Abordamos, finalmente, la conveniencia de considerar el papel de los esquemas cognitivos y, más extensamente, su conexión con los sistemas de valores, en la conformación de compren- siones consensuales de la tecnociencia compartidas por los miembros de grupo sociales específicos.

Para ello, hemos dividido este trabajo en cuatro partes. En esta primera sección, y tras estos considerandos introductorios, presentamos el estado de la cuestión del debate teórico sobre las posiciones críticas hacia la tecnociencia. En un segundo apartado, presentamos la metodología de investigación y los resultados obtenidos. A continuación, discutimos estos resultados poniéndolos en relación con las teorías planteadas previamente. Finalmente, establecemos las conclusiones de nuestra investigación, entre las que destacamos la creciente importancia de la ambivalencia en el análisis de la representación social de la ciencia y constatamos la influencia de los esquemas cognitivos en la configuración de esta ambivalencia en torno a aplicaciones controvertidas de la tecnociencia.

El papel que tiene la "comprensión pública de la ciencia" en la explicación de las actitudes hacia la tecnociencia ha generado debate y desacuerdo entre los estudiosos del tema, particularmente en su suposición de que los llamados temores "irracionales" hacia la tecnociencia entre algunos sectores de la opinión pública se basan en su falta de conocimiento científico. En un extremo del espectro están quienes creen en un modelo puramente de "déficit cognitivo" y sostienen que esa falta de conocimiento explica las posiciones críticas hacia la tecnociencia (Bodmer 1985; Ziman 1991); en el otro extremo, se sitúan quienes plantean que existen otros factores que explican esa falta de apoyo, así como quienes sostienen que el conocimiento científico es una construcción social, difícil de cuantificar (Johnson 1993).

Otras investigaciones sugieren que la respuesta se encuentra en algún punto intermedio (Brossard y Shanahan 2003). Cuando se trata de explicar la actitud del público hacia las innovaciones tecnológicas, otros factores han mostrado ser tan o más importantes que la "comprensión pública de la ciencia" y el conocimiento científico. Algunos estudios sugieren que el efecto de la confianza social en la percepción de tecnologías novedosas y potencialmente peligrosas (Siegrist et al. 2000; Priest 2001) o la confianza en los científicos, en las autoridades reguladoras y en la industria (Priest 2001) superan ampliamente la influencia del conocimiento científico. Sturgis y Allum (2004) señalan que los individuos con un mayor conocimiento político atribuyen los efectos indeseados a la influencia de la esfera económica sobre la actividad científica, no a los científicos ni a los políticos en general. Tampoco resulta satisfactorio el modelo del déficit cognitivo frente a evidencias como las aportadas por Scheufele et al. (2007), que muestran que los propios expertos nanocientíficos se muestran significativamente más preocupados que la población general sobre los impactos a largo plazo para la salud y el medio ambiente de la nanotecnología, a pesar de conocer mejor esa tecnología así como sus usos potenciales. 
Estudios recientes subrayan la importancia de los esquemas o atajos cognitivos en los posicionamientos ante aplicaciones tecnológicas controvertidas (Ho et al. 2008; Scheufele et al. 2009; Brossard et al. 2009). En concreto, diversos autores han puesto de manifiesto que mayores niveles de religiosidad se encuentran asociados a actitudes más críticas hacia nuevas aplicaciones tecnológicas (Gaskell et al. 2005; Brossard et al. 2009). Estos planteamientos se sitúan en línea con lo planteado por Dake (1991), cuando subrayó la importancia de las Weltanschauungen o visiones del mundo (worldviews) como "disposiciones orientadoras" que guían las respuestas de los individuos ante situaciones complejas. Asimismo, la teoría cultural plantea que los temores acerca de las nuevas tecnologías serían funcionales para el mantenimiento de las dinámicas culturales (Douglas y Wildavsky 1982). Según esta perspectiva, cada grupo social elige selectivamente qué supone un riesgo, poniendo de relevancia unos y restando importancia a otros, protegiendo ciertos patrones de comportamiento e interacción social frente a otros. De esta perspectiva se deriva que existiría una relación entre los sistemas de valores y la selección de los riesgos que manifiestan diferentes grupos sociales.

Esta perspectiva converge con la teorización de los esquemas o los atajos cognitivos. De manera cotidiana, los ciudadanos se enfrentan a cuestiones y debates sociales sobre los que tienen poca información o no tienen actitudes previas consolidadas pero una amplia mayoría logra habitualmente formarse una opinión en poco tiempo (Zaller 1992). Una explicación para esto es que muchos ciudadanos utilizan esquemas o atajos cognitivos (Downs 1957; Inglehart y Klingemann 1976; Fiske y Linville 1980; Sniderman et al. 1991), en lugar de dedicar tiempo y energía en obtener una cantidad elevada de información sobre el tema en cuestión, analizarla y sacar sus propias conclusiones. Estos esquemas pueden entenderse como "conocimiento previo organizado, abstraído de la experiencia concreta", que guía "el procesamiento de nueva información y la recuperación de la información almacenada" (Fiske y Linville 1980: 543). Los esquemas ideológicos se organizan en torno a categorías semánticas de alta significación, como el esquema izquierda-derecha ante debates políticos (Kumlin 2001).

Estos esquemas no poseen únicamente una dimensión individual, sino que tienen una correspondencia con el trasfondo social en el que las actitudes individuales surgen y se hacen explícitas. Para indagar sobre estos factores sociales, empleamos el concepto de "representaciones sociales", propuesto por el psicólogo social Serge Moscovici (1984), a partir de una reinterpretación del concepto durkheimiano de "representaciones colectivas". Como señala el propio Moscovici, la tecnociencia se ha convertido en la fuente más prolífica de creación de representaciones sociales del mundo moderno, por lo que el uso de este concepto en esta cuestión es, si cabe, más pertinente que ante cualquier otra. Este concepto de representación social permite "subrayar los factores sociales de la percepción y, con ello, destacar la importancia que los grupos y sus 'normas' tienen sobre la representación de la realidad social” (Torres Albero 2005a: 12).

En este trabajo nos ocupamos de analizar las representaciones sociales de la tecnociencia entre los españoles, así como de indagar el papel de los esquemas cognitivos de los individuos en sus posicionamientos y, más ampliamente, su conexión con los sistemas de valores en la conformación de las representaciones sociales de la tecnociencia compartidas por los miembros de grupos sociales específicos. Para ello, usamos los datos disponibles más recientes que aporta la Encuesta de Percepción Social de la Ciencia y la Tecnología 2014 (EPSCYT 2014), planteando la "pregunta de investigación primera": ‘es mayoritaria una representación social de la tecnociencia problematizada y ambivalente entre la población española?'. Nuestro trabajo parte de la hipótesis de que los esquemas ideológicos inciden en la formación de opinión ante aspectos controvertidos de la tecnociencia o sus nuevas aplicaciones. Estos esquemas ideológicos pueden estar asociados a distintos niveles de religiosidad, valores posmaterialistas o ideología política, entre otros aspectos. Para indagar sobre esta cuestión planteamos la "pregunta de investigación segunda”: ‘la relación entre conocimiento científico y confianza en aplicaciones tecnocientíficas controvertidas varía entre individuos con diferentes niveles de religiosidad, valores posmaterialistas o ideología política?' Así, los esquemas cognitivos vinculados a cada uno de estos aspectos permitirían inferencias y posicionamientos ante aplicaciones tecnocientíficas complejas, más allá del nivel del conocimiento o comprensión científica de los individuos.

\section{Metodología}

Como se ha indicado utilizamos los datos de la EPSCYT 2014, realizada por la Fundación Española para la Ciencia y la Tecnología (FECYT), para indagar con nuestras dos preguntas de investigación. El trabajo de campo consistió en 6355 entrevistas personales (cara a cara) y domiciliarias, realizadas entre el 14 de noviembre y el 20 de diciembre de 2014 a personas de 15 años de edad en adelante residentes en España durante cinco o más años ${ }^{1}$.

Para responder a la pregunta de investigación primera abordaremos dos niveles de análisis. En el primero, nos aproximamos a la imagen espontánea y general de la tecnociencia. Para ello, analizaremos las respuestas vertidas a las preguntas P1 y P2. La P1 se formuló de la siguiente manera en la encuesta referida: 'Cuando hablamos de ciencia, ¿qué le viene a la cabeza?', con respuesta abierta; y la P2: 'Si tuviera 
Ud. que hacer un balance de la ciencia y la tecnología teniendo en cuenta todos los aspectos positivos y negativos, ¿cuál de las siguientes opciones que le presento reflejaría mejor su opinión?', con las opciones de respuesta que se contemplan en la Tabla 2.

En un segundo nivel de análisis profundizamos en el análisis de estas representaciones sociales analizando tres preguntas (P3, P4 y P5) de la EPSCYT2014. La P3 refiere, 'Si tuviera que hacer el mismo balance sobre algunas aplicaciones concretas de la ciencia y la tecnología, teniendo en cuenta todos los aspectos positivos y negativos, ¿cuál de las siguientes opciones que le presento reflejaría mejor su opinión?'; La P4 plantea '¿Piensa que el progreso científico y tecnológico aporta más bien ventajas o más bien desventajas para...?'; y, finalmente, la P5 que consiste en una batería de valoración de varias afirmaciones en torno al contrato social de la tecnociencia. Los ítems de estas preguntas y sus opciones concretas de respuesta se contemplan, respectivamente, en las Tablas 3, 4 y 5 .

En un tercer nivel de análisis abordamos la pregunta de investigación segunda. Para ello, realizaremos una serie de regresiones logísticas multinomiales para cada una de las cuatro variables dependientes seleccionadas de la P3, correspondientes a las cuatro aplicaciones tecnocientíficas con un mayor grado de controversia entre la opinión pública española. En un segundo lugar, realizaremos una serie de regresiones logísticas binarias para las tres variables dependientes seleccionadas de la P4, correspondientes a los tres ítems que generan una mayor controversia entre la opinión pública acerca del impacto positivo o negativo que ha tenido la tecnociencia sobre ellos. Estos modelos nos ayudan a entender de qué manera cada una de las variables independientes influyen en la probabilidad de tener un posicionamiento crítico con cada una de las aplicaciones tecnocientíficas controvertidas analizadas, así como con el papel de la tecnociencia en los ámbitos de desarrollo social analizados, considerando simultáneamente los efectos de las otras variables independientes $y$, por tanto, determinando la significación e intensidad comparada de los efectos de cada variable en el resultado.

La medición de la religiosidad presenta cierta complejidad al ser la religión un concepto multidimensional que puede ser abordado de múltiples maneras. Existen diversas estrategias de medición de la dimensión religiosa presentes en la literatura que pueden agruparse en tres enfoques (Wald y Smidt 1993): un primer enfoque que aborda la dimensión religiosa como un conjunto de creencias; un segundo enfoque que la interpreta en su aspecto de "pertenencia"; y un tercer enfoque centrado en el "comportamiento", es decir, en la participación en actividades públicas o privadas de relevancia religiosa. Diversas encuestas, particularmente las del CIS, miden periódicamente el perfil religioso de los españoles en base a los enfoques de pertenencia y de participación religiosa. Los sucesivos sondeos muestran una alta identificación con la religión católica entre la población española, actualmente situada en torno al $72 \%-71.8 \%$ en el estudio 3124 del CIS de enero de 2016-. Sin embargo, en el caso español, esta "pertenencia" debe ser matizada con mediciones de comportamiento religioso, ya que la identificación con "lo católico" en España incluye actitudes religiosas dispares respecto a la práctica religiosa, desde la observación rigurosa hasta la indiferencia o, incluso, el rechazo de creencias centrales del catolicismo. Por este motivo, se plantea una escala de identificación con la práctica religiosa, a partir de la siguiente pregunta de la EPSCYT 2014: 'En el terreno religioso, ¿cómo se definiría usted?'. Para esta pregunta se ofrecen cinco opciones de respuesta: católico/a practicante; católico/a no practicante; perteneciente o practicante de otra religión; Indiferente o agnóstico/a; ateo/a. Esta escala permite establecer una "pertenencia" y, al mismo tiempo, una escala de "participación" religiosa para el $70 \%$ de españoles que se identifican como católicos. Medimos la religiosidad en función de la pertenencia a cuatro grupos: ateo/a, agnóstico/a, católico/a no practicante y católico/a practicante. En nuestra muestra, tan solo el $1,6 \%$ de los encuestados se ubica en otras opciones, por lo que optamos por no incluirlos en el análisis. Así, la escala de religiosidad que empleamos contempla cuatro categorías: 1=Ateo/a, 2=Agnóstico, 3=Católico no practicante, 4=Católico practicante. Por otro lado, estimamos la creencia general en la omnipotencia de la tecnociencia a partir de la siguiente afirmación: 'La ciencia y la tecnología pueden resolver cualquier problema', con tres categorías: $1=$ En desacuerdo (posición reflexiva), $2=\mathrm{Ni}$ de acuerdo ni en desacuerdo (posición indefinida), 3= De acuerdo (posición meliorativa). Finalmente, la medición de la ideología política se realiza a partir de la escala de posicionamiento ideológico para la que se han determinado cinco categorías: $1=$ =zquierda, 2=Centroizquierda, 3=Centro, 4=Centroderecha, $5=$ Derecha.

\section{RESULTAdOS Y DISCUSIÓN}

\section{Primer nivel de análisis: la imagen espontá- nea y general de la tecnociencia}

Cuando hablamos de representaciones sociales sobre la ciencia, el primer nivel de análisis pasa por indagar en las imágenes espontáneas que al respecto tiene la ciudadanía. Los resultados del primer nivel (preguntas P1 y P2) muestran una indiferenciación entre ciencia y tecnología, por un lado, y una imagen general positiva de la tecnociencia. Como reflejan los resultados de la EPSCYT 2014, esta imagen espontánea de la ciencia está vinculada con una gran diversidad de elementos. 
En la Tabla 1 se muestran los resultados diferenciados que han sido mencionados por, al menos, el $3 \%$ de los encuestados en la $\mathrm{P} 1$; el resto de menciones se agrupan bajo el genérico de 'Otros'. Los principales aspectos con los que se relaciona de manera espontánea a la ciencia son la innovación y la investigación $(32 \%)$, la medicina, la salud y los tratamientos médicos $(27 \%)$, los laboratorios y la experimentación $(20 \%)$ y con las ramas científicas de biología, química o física (14\%). El alto porcentaje de la categoría 'Otros' $(29 \%)$ da cuenta de las variopintas respuestas vertidas por los encuestados que, sin superar el referido umbral del $3 \%$, indican sobre todo referencias a disciplinas o aplicaciones tecnocientíficas específicas, pero también menciones al futuro y al progreso $(2 \%)$. Finalmente, la ciencia es asociada con algo 'difícil de entender' por un $5,3 \%$ de los encuestados. El análisis de esta última respuesta permite constatar que, a menor edad y a mayor nivel de estudios, esta proporción disminuye considerablemente -hasta el 2,6\% entre los menores de 34 años y hasta el $1,4 \%$ entre quienes tienen estudios universitarios-.

La naturaleza de la diversidad de respuestas vertidas -desde disciplinas específicas hasta aplicaciones concretas, pasando por los laboratorios y la carrera espacial- apunta a que la mayor parte de los individuos no establecen una diferencia clara, en términos generales, entre ciencia y tecnología a pesar de que la pregunta se refería explícita y exclusivamente a la ciencia. De hecho, en los estudios sociales de ciencia y tecnología es frecuente el uso del término tecnociencia (Bachelard 1953), que se refiere al entramado indisoluble entre las ciencias y las

Tabla 1.

La imagen espontánea de la ciencia (en porcentajes). P1. Cuando hablamos de ciencia, ¿qué le viene a la cabeza? (máximo 2 resp.).

\begin{tabular}{|l|c|}
\hline & TOTAL \\
\hline BASE & 6355 \\
\hline Innovación/investigación & 32,2 \\
\hline Medicina/salud/tratamientos & 27,2 \\
\hline Laboratorios/experimentación & 20,2 \\
\hline Biología/química/física & 14,4 \\
\hline Genética/ADN & 7,8 \\
\hline Ordenadores/tecnología & 7,6 \\
\hline Nuevas aplicaciones tecnológicas & 5,3 \\
\hline Difícil de entender & 5,3 \\
\hline Ingenierías & 4,3 \\
\hline Importante/necesario & 3,8 \\
\hline Astronomía/espacio/carrera espacial & 3,5 \\
\hline Otros & 28,7 \\
\hline No sabe / No contesta & 4,9 \\
\hline
\end{tabular}

Fuente: EPSCYT 2014. tecnologías contemporáneas en contraposición a las concepciones tradicionales. Los datos de la encuesta indican que esta concepción conjunta de ciencia y tecnología también está presente entre la opinión pública a la hora de abordar lo que se entiende por ciencia. En este sentido, no parece sostenible, como ya hemos señalado anteriormente (Torres-Albero 2005a), la tesis tradicional del enfoque de la comprensión pública de la ciencia que defiende que la tecnociencia tiene una mayoritaria valoración positiva entre la opinión pública de las sociedades desarrolladas, y que, en todo caso, la valoración negativa quedaría para la tecnología; sino que, por el contrario, se trataría de aspectos prácticamente indiferenciados por parte de la opinión pública.

La Tabla 2 ofrece los resultados de la P2, midiendo el balance que hacen los entrevistados entre los beneficios y los perjuicios de la tecnociencia, teniendo en cuenta todos sus aspectos. Observamos un balance mayoritariamente positivo entre los beneficios y perjuicios de la ciencia y la tecnología $(59,5 \%)$. Sin embargo, como en encuestas anteriores, se registran opiniones que no manifiestan una valoración positiva. A pesar de que esta pregunta tiene una formulación general $-y$ de que su análisis debe ser completado con otras preguntas planteadas en los siguientes niveles de análisis- se pueden constatar aquí posiciones alejadas de la posición ilustrada que vincula lineal y positivamente tecnociencia y progreso. Estas posiciones críticas se expresan, en su mayor parte, con la formulación de ambivalencia equidistante, que considera que los beneficios y los perjuicios de la ciencia y la tecnología estarían equilibrados.

Tabla 2.

Balance general de la ciencia y la tecnología (en porcentajes).

P2. Si tuviera Ud. que hacer un balance de la ciencia y la tecnología teniendo en cuenta todos los aspectos positivos y negativos, ¿cuál de las siguientes opciones que le presento reflejaría mejor su opinión?

\begin{tabular}{|l|c|}
\hline & TOTAL \\
\hline BASE & 6355 \\
\hline $\begin{array}{l}\text { Los beneficios de la ciencia y la tecnolo- } \\
\text { gía son mayores que sus perjuicios }\end{array}$ & 59,5 \\
\hline $\begin{array}{l}\text { Los beneficios y los perjuicios de la cien- } \\
\text { cia y tecnología están equilibrados }\end{array}$ & 26,1 \\
\hline $\begin{array}{l}\text { Los perjuicios de la ciencia y la tecnología } \\
\text { son mayores que los beneficios }\end{array}$ & 5,3 \\
\hline $\begin{array}{l}\text { No tengo una opinión formada sobre esta } \\
\text { cuestión }\end{array}$ & 6,9 \\
\hline No contesta & 2,1 \\
\hline
\end{tabular}

Fuente: EPSCYT 2014. 
En conjunto, algo más del $40 \%$ no expresa una imagen positiva de la tecnociencia ante esta pregunta. La mayor parte de estas respuestas (26\%) sitúan en un equilibrio los beneficios y los perjuicios, mientras que el $5 \%$ muestra una posición crítica de este balance, el $7 \%$ asegura que no tiene una opinión formada y el $2 \%$ prefiere no dar una opinión. Diversos estudios apuntan a la existencia de un contínuum actitudinal hacia la tecnociencia (Bauer et al. 2000; Torres-Albero 2009) con un espacio central que no sería neutral sino ambivalente (Martinez et al. 2005). Esta constatación de un cierto nivel de ambivalencia en la valoración general de la tecnociencia deja entrever ya que esta evaluación de la tecnociencia no es aproblemática. El contínuum actitudinal sobre el que se expresan las representaciones de la tecnociencia no puede ser analizado únicamente a partir de esta pregunta general que, a partir de una escala de tres opciones, permite identificar únicamente la ambivalencia equidistante. En el análisis de aspectos más concretos nos permitirá describir con más precisión las representaciones sociales de la tecnociencia.

\section{Segundo nivel de análisis: profundización de la ambivalencia y problematización de la tecnociencia}

Si bien la imagen espontánea de la tecnociencia está, fundamentalmente, relacionada con significados positivos entre la población, su valoración general muestra a una parte considerable de la población que no asume que los beneficios de la tecnociencia superan sus desventajas. Esta valoración general se va delineando y problematizando ante cuestiones específicas, hasta observarse posiciones críticas entre una mayoría de la población ante ciertas aplicaciones. En este segundo nivel de análisis, como se ha indicado, hemos realizado un análisis univariante de las preguntas P3, P4 y P5.

La P3 indaga sobre el balance entre beneficios y perjuicios que realizan los encuestados sobre una serie de aplicaciones concretas de la ciencia y la tecnología: el cultivo de plantas modificadas genéticamente, la clonación, la energía nuclear, la investigación con células madre, el fracking, Internet, la telefonía móvil, los aerogeneradores (molinos de viento) y el diagnóstico genético de enfermedades. Mientras que algunas aplicaciones tecnológicas son claramente valoradas como positivas por la mayoría -el diagnóstico genético de enfermedades (82\%), la investigación con células madre $(77 \%)$, los aerogeneradores $(76 \%)$, Internet $(69 \%)$ y la telefonía móvil (65\%)-, otras son valoradas negativamente -la energía nuclear (54\%), la clonación (43\%) y el cultivo de plantas modificadas genéticamente (42\%)-, como se observa en la siguiente tabla. Es igualmente destacable el desconocimiento de la tecnología de la fractura hidráulica (fracking): hasta el $57 \%$ reconoce no conocer esta aplicación o no tener una opinión formada sobre el tema (Tabla 3). Observamos, así, que no todas las aplicaciones tecnocientíficas son valoradas positivamente. La mayoría considera que no toda aplicación tecnocientífica es positiva ni conlleva inevitablemente el progreso, sino que algunas aplicaciones son más perjudiciales que beneficiosas. La tecnociencia no es considerada, así, como un todo benefactor, sino que la gran mayoría de los individuos discrimina entre sus aplicaciones concretas.

Tabla 3.

Balance sobre algunas aplicaciones concretas de la ciencia y la tecnología (en porcentajes). P3. "Si tuviera que hacer el mismo balance sobre algunas aplicaciones concretas de la ciencia y la tecnología, teniendo en cuenta todos los aspectos positivos y negativos, ¿cuál de las siguientes opciones que le presento reflejaría mejor su opinión?"

\begin{tabular}{|l|c|c|c|c|c|c|}
\hline $\begin{array}{l}\text { Aplicaciones } \\
\text { tecnocientíficas }\end{array}$ & $\begin{array}{l}\text { Beneficios } \\
\text { superan a los } \\
\text { perjuicios }\end{array}$ & $\begin{array}{l}\text { Beneficios y } \\
\text { perjuicios están } \\
\text { equilibrados }\end{array}$ & $\begin{array}{l}\text { Los perjuicios } \\
\text { son mayores que } \\
\text { los beneficios }\end{array}$ & $\begin{array}{l}\text { No sé qué } \\
\text { es esta } \\
\text { aplicación }\end{array}$ & $\begin{array}{l}\text { No tengo una } \\
\text { opinión }\end{array}$ & $\begin{array}{l}\text { No } \\
\text { contesta }\end{array}$ \\
\hline La energía nuclear & 16,4 & 19,8 & 54,4 & 1,9 & 7,1 & 0,3 \\
\hline La clonación & 18,7 & 20,2 & 42,7 & 4,0 & 14,0 & 0,4 \\
\hline $\begin{array}{l}\text { Cultivo de plantas } \\
\text { modificadas genéticamente }\end{array}$ & 17,3 & 24,1 & 41,7 & 4,1 & 12,5 & 0,4 \\
\hline El fracking & 7,0 & 10,2 & 24,5 & 42,7 & 14,4 & 1,2 \\
\hline $\begin{array}{l}\text { El diagnóstico genético de } \\
\text { enfermedades }\end{array}$ & 82,2 & 10,3 & 2,5 & 1,1 & 3,7 & 0,2 \\
\hline Los molinos de viento & 75,7 & 15,6 & 3,8 & 1,0 & 3,5 & 0,0 \\
\hline $\begin{array}{l}\text { La investigación con células } \\
\text { madre }\end{array}$ & 75,4 & 13,0 & 4,3 & 2,4 & 4,7 & 0,2 \\
\hline La telefonía móvil & 67,1 & 24,8 & 6,0 & 0,4 & 1,6 & 0,2 \\
\hline Internet & 65,8 & 24,6 & 4,8 & 0,8 & 3,8 & 0,0 \\
\hline
\end{tabular}

Fuente: EPSCYT 2014. 
En suma, la mayoría de los ciudadanos considera que no toda aplicación tecnocientífica es necesariamente positiva sino que algunas aplicaciones son más perjudiciales que beneficiosas. Entre la población española, alguna de las aplicaciones que podríamos considerar como más polémicas son la energía nuclear, la clonación y el cultivo de plantas modificadas genéticamente.

La P4 indaga sobre la valoración de la tecnociencia para diversos ámbitos de la sociedad, preguntando al entrevistado que considere si 'el progreso científico y tecnológico aporta más bien ventajas o más bien desventajas' para los siguientes aspectos: el desarrollo económico, la calidad de vida en la sociedad, la seguridad y la protección de la vida humana, la conservación del medio ambiente y la naturaleza, hacer frente a las enfermedades y epidemias, los productos de alimentación y la producción agrícola, la generación de nuevos puestos de trabajo, el incremento y mejora de las relaciones entre las personas, el aumento de las libertades individuales, y la reducción de diferencias entre países ricos y pobres. En primer lugar, observamos diferencias significativas entre distintos ámbitos. La contribución a la mejora de la salud (hacer frente a enfermedades y epidemias), como en el apartado anterior, destaca por ser el ámbito que mayor consenso aglutina entre la población $(94,6 \%)$ en un sentido positivo. Una amplia mayoría también considera positivo también el impacto de la tecnociencia en la mejora de la calidad de vida en la sociedad $(86,6 \%)$, en el desarrollo económico $(85,4 \%)$, así como en la seguridad y la protección de la vida humana $(82,1 \%)$.
En cambio, se constata un mayor disenso cuando se valora su aportación a la conservación del medio ambiente, la mejora de las relaciones interpersonales, las libertades individuales, así como la reducción de las diferencias entre países ricos y pobres. Como se observa en la tabla siguiente, quienes valoran que la tecnociencia aporta más desventajas ante estas cuestiones oscilan entre el $46,7 \%$ y el $27,7 \%$, según el caso considerado (Tabla 4). Resulta llamativo que todas estas cuestiones están vinculadas con valores que podemos asociar con el posmaterialismo (Inglehart 1995) conservación del medio ambiente, relaciones interpersonales, libertad, solidaridad. Por el contrario, la contribución de la tecnociencia ante aquellas cuestiones asociadas con los valores materialistas tradicionales, como la seguridad, la economía, la calidad de vida y la salud, reciben una valoración positiva por parte de una amplia mayoría entre la opinión pública.

En diversos estudios se ha analizado la relación de los valores posmaterialistas con las actitudes hacia la tecnociencia, poniendo de manifiesto su complejidad (Van Deth y Scarbrough 1995). Por un lado, se ha observado que las personas con valores posmaterialistas son propensas a la innovación, así como a la organización racional y científica de la vida social; pero por otro, muestran actitudes críticas hacia ciertas aplicaciones tecnocientíficas, como la energía nuclear, así como hacia el impacto negativo que la actividad humana tiene sobre el entorno natural (Gabriel y Van Deth 1995).

Tabla 4.

Valoración del progreso científico y tecnológico para diferentes dimensiones sociales (en porcentajes). P.4 "Le voy a mostrar una serie de aspectos. ¿Piensa que el progreso científico y tecnológico aporta más bien ventajas o más bien desventajas para..."

\begin{tabular}{|l|c|c|c|}
\hline Aspectos & Ventajas & Desventajas & NS/NC \\
\hline El desarrollo económico & 85,4 & 13,8 & 0,8 \\
\hline La calidad de vida en la sociedad & 86,6 & 12,7 & 0,7 \\
\hline La seguridad y la protección de la vida humana & 82,1 & 17,0 & 0,8 \\
\hline La conservación del medio ambiente y la naturaleza & 66,7 & 32,3 & 0,9 \\
\hline Hacer frente a las enfermedades y epidemias & 94,6 & 4,8 & 0,6 \\
\hline Los productos de alimentación y la producción agrícola & 68,9 & 30,2 & 0,9 \\
\hline La generación de nuevos puestos de trabajo & 68,2 & 30,7 & 1,1 \\
\hline El incremento y mejora de las relaciones entre las personas & 62,9 & 35,6 & 1,5 \\
\hline El aumento de las libertades individuales & 62,2 & 36,0 & 1,8 \\
\hline La reducción de diferencias entre países ricos y pobres & 47,3 & 51,4 & 1,3 \\
\hline
\end{tabular}

Fuente: EPSCYT 2014. 
La P5 nos ofrece una batería de cuestiones ordenada por pares lógicos de lo que podemos denominar como el contrato social con la tecnociencia. La escala Likert de cinco opciones presente en esta batería de preguntas nos permite observar en mejores condiciones el contínuum actitudinal. Como muestra la Tabla 5, las posiciones intermedias ('Bastante de acuerdo', 'Ni de acuerdo ni en desacuerdo'; 'Bastante en desacuerdo') son mayoritarias ante las diferentes cuestiones asociadas al contrato social de la tecnociencia. El análisis de estos pares de preguntas nos ayuda a perfilar cuánto y cómo se ha modificado en la opinión pública el contrato social implícito a favor de la ciencia (Blanco e Iranzo 2000) debido a su problematización.

El primer par de preguntas constata una cierta desconfianza ante la neutralidad de la actividad tecnocientífica. Una proporción significativa de la población $(38 \%)$ recela de la influencia de intereses privados, a través de su financiación, sobre los resultados publicados de las investigaciones, y un $26 \%$ se manifiesta 'ni de acuerdo ni en desacuerdo' ante esta cuestión.
Por otro lado, el 34\% se muestra 'muy de acuerdo' o 'bastante de acuerdo' con que los investigadores y los expertos no permiten que quienes les financian influyan en los resultados de sus investigaciones.

El segundo par de afirmaciones resulta, asimismo, revelador acerca del cambio en las características del contrato social con la tecnociencia y, en general, de su representación social, ya que confronta la concepción ilustrada tradicional de la tecnociencia. Para buena parte de la población (45\%) la tecnociencia ya no es la panacea, como se derivaba de la visión ilustrada; solo el $26 \%$ cree hoy que la tecnociencia puede resolver cualquier problema. Esta pregunta nos sirve de indicador en próximos análisis de creencia en el carácter meliorativo de la tecnociencia, por un lado, o de una posición reflexiva, por otro. En los otros extremos, el 21,9\% se muestra 'Muy en desacuerdo' con la omnipotencia tecnocientífica y el $35,4 \%$ con su capacidad para explicarlo todo. Las diferencias entre grupos sociales ante esta posición son bajas y presentan un relativo consenso transversal entre los diferentes colectivos.

Tabla 5.

Dimensiones específicas del "contrato social" de la tecnociencia (en porcentajes).

\begin{tabular}{|c|c|c|c|c|c|c|c|}
\hline Afirmaciones & $\begin{array}{l}\text { Muy de } \\
\text { acuerdo }\end{array}$ & $\begin{array}{l}\text { Bastante } \\
\text { de acuerdo }\end{array}$ & $\begin{array}{l}\text { Ni de } \\
\text { acuerdo ni } \\
\text { en desac. }\end{array}$ & $\begin{array}{l}\text { Bastante en } \\
\text { desacuerdo }\end{array}$ & $\begin{array}{c}\text { Muy en } \\
\text { desacuerdo }\end{array}$ & NS/NC & Media \\
\hline $\begin{array}{l}\text { No podemos confiar en que los científicos } \\
\text { digan la verdad sobre temas controvertidos } \\
\text { debido a que dependen más y más de la } \\
\text { financiación de la industria }\end{array}$ & 14,5 & 23,5 & 26,4 & 18,3 & 8,6 & 8,8 & 3,19 \\
\hline $\begin{array}{l}\text { Los investigadores y los expertos no } \\
\text { permiten que quienes les financian influyan } \\
\text { en los resultados de sus investigaciones }\end{array}$ & 9,7 & 24,0 & 28,4 & 17,3 & 10,4 & 10,2 & 3,06 \\
\hline $\begin{array}{l}\text { La ciencia y la tecnología pueden resolver } \\
\text { cualquier problema }\end{array}$ & 5,1 & 20,4 & 25,1 & 23,3 & 21,9 & 4,2 & 2,62 \\
\hline $\begin{array}{l}\text { Siempre habrá cosas que la ciencia no } \\
\text { podrá explicar }\end{array}$ & 35,4 & 35,4 & 15,1 & 8,0 & 3,0 & 3,1 & 3,95 \\
\hline $\begin{array}{l}\text { Es erróneo imponer restricciones a } \\
\text { las nuevas tecnologías hasta que se } \\
\text { demuestre científicamente que pueden } \\
\text { causar daños graves a los seres humanos } \\
\text { y al medio ambiente }\end{array}$ & 10,8 & 19,7 & 24,4 & 21,7 & 15,9 & 7,4 & 2,87 \\
\hline $\begin{array}{l}\text { Mientras se desconozcan las consecuencias } \\
\text { de una nueva tecnología, se debería actuar } \\
\text { con cautela y controlar su uso para proteger } \\
\text { la salud y el medio ambiente }\end{array}$ & 39,7 & 36,1 & 13,6 & 4,5 & 1,3 & 4,8 & 4,14 \\
\hline $\begin{array}{l}\text { Los conocimientos científicos son la mejor } \\
\text { base para elaborar leyes y regulaciones }\end{array}$ & 8,2 & 22,7 & 34,6 & 14,8 & 9,0 & 10,6 & 3,07 \\
\hline $\begin{array}{l}\text { En la elaboración de leyes y regulaciones, } \\
\text { los valores y las actitudes son tan } \\
\text { importantes como los conocimientos } \\
\text { científicos }\end{array}$ & 20,0 & 32,0 & 28,2 & 8,0 & 2,8 & 9,0 & 3,64 \\
\hline $\begin{array}{l}\text { Las decisiones sobre la ciencia y la } \\
\text { tecnología es mejor dejarlas en manos de } \\
\text { los expertos }\end{array}$ & 32,3 & 36,3 & 18,6 & 6,9 & 2,4 & 3,5 & 3,93 \\
\hline $\begin{array}{l}\text { Los ciudadanos deberían desempeñar un } \\
\text { papel más importante en las decisiones } \\
\text { sobre ciencia y tecnología }\end{array}$ & 22,5 & 30,6 & 25,3 & 13,5 & 2,7 & 5,4 & 3,60 \\
\hline
\end{tabular}

Fuente: EPSCYT 2014. 
Asimismo, una amplia mayoría $(75,8 \%)$ considera que, 'mientras se desconozcan las consecuencias de una nueva tecnología, se debería actuar con cautela y controlar su uso para proteger la salud y el medio ambiente'. Solamente el $1,3 \%$ se muestra totalmente contrario a la aplicación del principio de precaución y el $4,5 \%$ algo contrario. Hay que tener en cuenta para la interpretación de esta pregunta que las proporciones cambian sensiblemente con la formulación de la pregunta: el uso de las expresiones 'imponer restricciones' y 'erróneo' reduce los porcentajes, así como el efecto de optar por el 'desacuerdo', vinculado con la deformación conservadora observada en los estudios de opinión pública.

Por otro lado, se puede observar que el cientifismo no reúne una alta adhesión entre la población. Apenas el $8,2 \%$ se muestra totalmente de acuerdo con que 'los conocimientos científicos son la mejor base para elaborar leyes y regulaciones'. Y solamente el $10,8 \%$ se muestra muy o bastante en desacuerdo con que 'en la elaboración de leyes y regulaciones, los valores y las actitudes son tan importantes como los conocimientos científicos'.

Finalmente, una mayoría de los entrevistados $(68,2 \%)$ creen en las bondades de la tecnocracia, pero también una mayoría $(53,1 \%)$ confía en la participación ciudadana en las decisiones sobre ciencia y tecnología. A tenor de los resultados, no parecen ser dos opciones excluyentes entre muchos entrevistados, sino que parecen estar a favor de que aumente la participación ciudadana en las decisiones sobre ciencia y tecnología al mismo tiempo que los expertos sigan teniendo un papel preponderante. De hecho, solo el 19\% de quienes están de acuerdo o muy de acuerdo con que 'las decisiones sobre la ciencia y la tecnología es mejor dejarlas en manos de los expertos' se muestran en desacuerdo con que 'los ciudadanos deberían desempeñar un papel más importante en las decisiones sobre ciencia y tecnología'. Las respuestas de la primera pregunta parecen apuntar a la voluntad de que los políticos y los poderes económicos no condicionen a los expertos en lo que se refiere a su trabajo; en cambio, la segunda pregunta apunta a la voluntad de una mayor participación ciudadana en las decisiones sobre ciencia y tecnología, sin que ambas opciones sean contradictorias para una mayoría de los individuos.

\section{Tercer nivel de análisis: los factores determi- nantes de las representaciones sociales}

Hasta aquí hemos constatado que la ambivalencia y la problematización ocupan un papel central en la caracterización de la representación social de la tecnociencia mayoritaria entre la población española. Se observan disensos entre grupos de población en su valoración de la tecnociencia y la representación mayoritaria se caracteriza por no ser uniforme: en unas mismas personas, se obser- van posicionamientos de signo distinto acerca de diferentes aspectos de la tecnociencia.

Nos interesa ahora identificar si los esquemas cognitivos que permiten a los individuos establecer posicionamientos en torno a nuevas aplicaciones tecnocientíficas aparecen vinculados con la religiosidad, la ideología política y la tecnológica, controlando el efecto del nivel educativo, además de por edad, sexo y nivel de ingresos. Para ello consideramos dos bloques de variables dependientes: el balance sobre aplicaciones tecnocientíficas controvertidas (P3) y la valoración del progreso científico y tecnológico para diferentes dimensiones sociales (P4).

Para el primer bloque de variables dependientes, tomamos las referidas a las tres aplicaciones con peor valoración -la energía nuclear, la clonación y el cultivo de plantas modificadas genéticamente- y una cuarta que presenta un alto nivel de desconocimiento entre la población -el fracking- mediante sendos análisis de regresión logística multinomial, con el sexo, la edad, el nivel educativo, la religiosidad, la ideología política, la ideología tecnocientífica (referida a la creencia en la omnipotencia de la tecnociencia) y el nivel de ingresos como variables independientes. En la tabla 6 se muestran los parámetros resultantes de las pruebas realizadas.

En primer lugar, constatamos que la influencia de las variables de carácter ideológico: en general, para las aplicaciones más controvertidas -nuclear, clonación y OGM- se observan posiciones más críticas en las posiciones más a la izquierda en la escala ideológica, entre quienes tienen una menor creencia en la omnipotencia de la tecnociencia y entre los menores niveles de religiosidad. Esta observación general encuentra dos excepciones: por un lado, el efecto de la religiosidad ante los OGM se astringe cuando se controla por las otras dos variables ideológicas y, por otro, ante la clonación las posiciones más centradas del arco ideológico y las de menor religiosidad son las menos críticas. La explicación para estas excepciones apunta a la manera en la que se ha desarrollado el debate público en estas cuestiones y qué tipo de asociaciones -religiosas, ecologistas o políticasse han posicionado críticamente.

El efecto del nivel educativo es únicamente significativo en el caso de la energía nuclear y no anula el efecto del resto de variables mencionadas. La creencia general en la omnipotencia de la tecnociencia actúa sobre la percepción benévola de las tres aplicaciones más controvertidas. Ante la aplicación con mayor desconocimiento público -fracking- aquellas personas posicionadas críticamente lo hacen fundamentalmente siguiendo un esquema ideológico en el eje izquierda-derecha. Por otra parte, es interesante observar el efecto de la religiosidad, ya que no actúa en una misma dirección en España y Estados Unidos, ni en todas las aplicaciones tecnocientíficas. Religiosidad y representaciones sociales de la tec- 
Tabla 6.

Resumen de los resultados de las regresiones logísticas multinomiales (P3).

\begin{tabular}{|c|c|c|c|c|c|c|c|c|c|}
\hline \multirow{2}{*}{\multicolumn{2}{|c|}{ Los perjuicios son mayores que los beneficios... }} & \multicolumn{2}{|c|}{ Energía nuclear } & \multicolumn{2}{|c|}{ Clonación } & \multicolumn{2}{|c|}{ OGM } & \multicolumn{2}{|c|}{ Fracking } \\
\hline & & Sig. & $\operatorname{Exp}(B)$ & Sig. & $\operatorname{Exp}(B)$ & Sig. & $\operatorname{Exp}(B)$ & Sig. & $\operatorname{Exp}(B)$ \\
\hline \multicolumn{2}{|l|}{ Intersección } & ,289 & &, 000 & & ,155 & & ,646 & \\
\hline Sexo & Hombre (ref. mujer) & 000 & 671 & 944 & 1,007 & ,117 & 847 &, 002 & ,616 \\
\hline \multirow{3}{*}{ Edad } & 15 a 34 años & 046 & ,744 & ,002 & 661 & 104 & ,795 & ,000 & ,424 \\
\hline & 35 a 54 años & , 148 & ,815 & ,046 & ,772 & ,633 & ,939 & ,022 & 643 \\
\hline & 55 años y más (ref) & & & & & & & & \\
\hline \multirow{3}{*}{ Nivel educativo } & Terciaria & ,002 & ,620 & ,157 & ,820 & ,504 & 1,105 & ,741 & 1,068 \\
\hline & Secundaria & 048 & ,767 & ,443 & 1,102 & 844 & ,975 & 120 & 1,319 \\
\hline & Primaria (ref) & & & & & & & & \\
\hline \multirow{4}{*}{ Religiosidad } & Ateo & ,008 & 1,763 & ,005 & ,565 & 925 & ,981 & ,831 & ,936 \\
\hline & Agnóstico & ,002 & 1,861 &, 000 & ,409 & ,818 & ,955 &, 572 & 844 \\
\hline & Católico no-practicante & ,447 & 1,132 & 071 & ,738 & 652 & ,929 & ,238 & ,733 \\
\hline & Católico practicante (ref) & & & & & & & & \\
\hline \multirow{3}{*}{$\begin{array}{l}\text { La tecnociencia puede } \\
\text { resolver cualquier } \\
\text { problema }\end{array}$} & En desacuerdo & 000 & 1,701 & ,001 & 1,500 & ,000 & 1,968 & ,234 & 1,232 \\
\hline & Ni de acuerdo ni en des. & ,240 & 1,183 & ,132 & 1,226 &, 134 & 1,231 & ,994 & 1,001 \\
\hline & De acuerdo (ref) & & & & & & & & \\
\hline \multirow{5}{*}{ Ideología política } & Izquierda & ,004 & 2,329 & ,177 & 1,457 & ,002 & 2,541 & ,000 & 8,888 \\
\hline & Centroizquierda & ,000 & 2,808 & ,007 & 1,674 & ,000 & 2,125 & ,000 & 4,068 \\
\hline & Centro & ,000 & 2,204 & ,031 & 1,516 & ,000 & 1,989 & ,000 & 3,616 \\
\hline & Centroderecha & ,002 & 1,814 &, 435 & 1,166 &, 012 & 1,638 &, 002 & 2,382 \\
\hline & Derecha (ref) & & & & & & & & \\
\hline \multirow{5}{*}{$\begin{array}{l}\text { Nivel de ingresos } \\
\text { familiares }\end{array}$} & Más de $2400 €$ & ,082 & ,646 & ,000 & ,327 & ,698 & 1,099 & ,599 & 1,190 \\
\hline & Entre 1500 y $2004 €$ & ,096 & 1,457 & ,011 & ,552 & 108 & 1,405 & 299 & 1,361 \\
\hline & Entre 1000 y $1500 €$ & ,036 & 1,592 & ,832 & 1,051 & ,000 & 2,399 & ,088 & 1,662 \\
\hline & Entre 700 y $1000 €$ & 027 & 1,729 & ,926 & 1,024 & ,002 & 2,079 & ,055 & 1,899 \\
\hline & Menos de $700 €$ (ref) & & & & & & & & \\
\hline \multicolumn{2}{|l|}{$-2 \log$ de verosimilitud } & \multicolumn{2}{|c|}{4075,325} & \multicolumn{2}{|c|}{3520,286} & \multicolumn{2}{|c|}{4490,581} & \multicolumn{2}{|c|}{2593,575} \\
\hline \multicolumn{2}{|l|}{$\mathrm{R}^{2}$ Nagelkerke } & \multicolumn{2}{|c|}{111} & \multicolumn{2}{|c|}{,092 } & \multicolumn{2}{|c|}{,087 } & \multicolumn{2}{|c|}{,121 } \\
\hline
\end{tabular}

Nota: La categoría de referencia de las variables dependientes es 'Los beneficios superan a los perjuicios'.

nociencia ya se han mostrado asociadas en otros estudios, como hemos señalado. Sin embargo, las observaciones que presentamos acerca de la influencia de la religiosidad en la valoración de aplicaciones tecnocientíficas controvertidas son consistentes únicamente de manera parcial con los resultados de estudios previos.

Así, en Estados Unidos, los individuos con un mayor nivel de religiosidad son más propensos a oponerse a las aplicaciones tecnocientíficas (Gaskell et al. 2005: 1909) a las que perciben como alteradoras de la naturaleza y los procesos naturales, mientras que en España son los menos propensos a oponerse -con la excepción de la clonación ${ }^{2}$. Estos contrastes indicarían que no hay algo esencial en la religiosidad que apunte en una dirección específica en su relación con la tecnociencia -como sugieren otros estudios-, sino que el nivel de religiosidad sirve de indicador para discriminar representaciones sociales opuestas ante la tecnociencia que se construyen en diferentes grupos. Así, la extensión de ciertas creen- cias, como el creacionismo, entre personas con altos niveles de religiosidad en Estados Unidos muy probablemente contribuye a vincular la religiosidad a esquemas cognitivos que penalizan la manipulación de los procesos naturales por el ser humano; mientras que en otras culturas, como España, la religiosidad parece haberse asociado con una heurística más confiada en la intervención de la tecnociencia. Esto contribuiría a explicar el sentido diverso que puede adoptar el heurístico religioso en diversas culturas.

Para profundizar en la comprensión de la relación entre estas variables y los sistemas de valores, analizamos las respuestas a la batería de preguntas P4 (ver Tabla 4), en la que podemos identificar áreas de impacto de la tecnociencia estrechamente vinculadas con valores materialistas (Inglehart 1995) -como el desarrollo económico, la calidad de vida en la sociedad, hacer frente a las enfermedades y epidemias, o la generación de nuevos puestos de trabajo- y otras con valores posmaterialistas -como la conservación del medio ambiente y la naturaleza 
(ecología), el aumento de las libertades individuales (libertad), y la reducción de diferencias entre países ricos y pobres (solidaridad). Realizamos regresiones logísticas binarias para los tres ítems que generan una mayor controversia entre la opinión pública que, como hemos visto, son aquellos ítems vinculados en mayor medida con valores posmaterialistas.

Los resultados constatan la relación inversa entre el nivel de religiosidad con la crítica del impacto de la tecnociencia sobre los ámbitos posmaterialistas (Tabla 7). Así, los mayores niveles de religiosidad estarían asociados con los valores materialistas, más que con los posmaterialistas; mientras que los menores niveles de religiosidad están asociados en mayor medida con preocupaciones asociadas a valores posmaterialistas. Asimismo, se constatan las relaciones entre estas preocupaciones y la creencia general en la omnipotencia de la tecnociencia; la ideología política, por su parte, reduce su capacidad explicativa ante estas preocupaciones, mantenién- dose su influencia únicamente sobre las preocupaciones acerca de las libertades individuales.

En un segundo modelo, añadimos la variable de ingresos familiares para testar la influencia de los condicionantes económicos en la adhesión a los valores posmateriales que muestran los individuos una relación apuntada por Inglehart (1995) pero contestada desde diferentes perspectivas. Los resultados de las regresiones muestran que tanto el nivel de religiosidad, como la ideología política y tecnológica mantienen una capacidad predictiva elevada cuando se controla su efecto por el nivel de ingresos familiares, mientras que el efecto del nivel de ingresos familiares aparece desdibujado en los pocos casos que se muestra significativo estadísticamente. Estos resultados apuntan, en un sentido similar a lo señalado por Betz (1990) y De Graaf y Evans (1996), que los sistemas de valores posmaterialistas en torno a la tecnociencia estarían relacionados principalmente con factores no económicos.

Tabla 7.

Resultados de las regresiones logísticas binarias (P4).

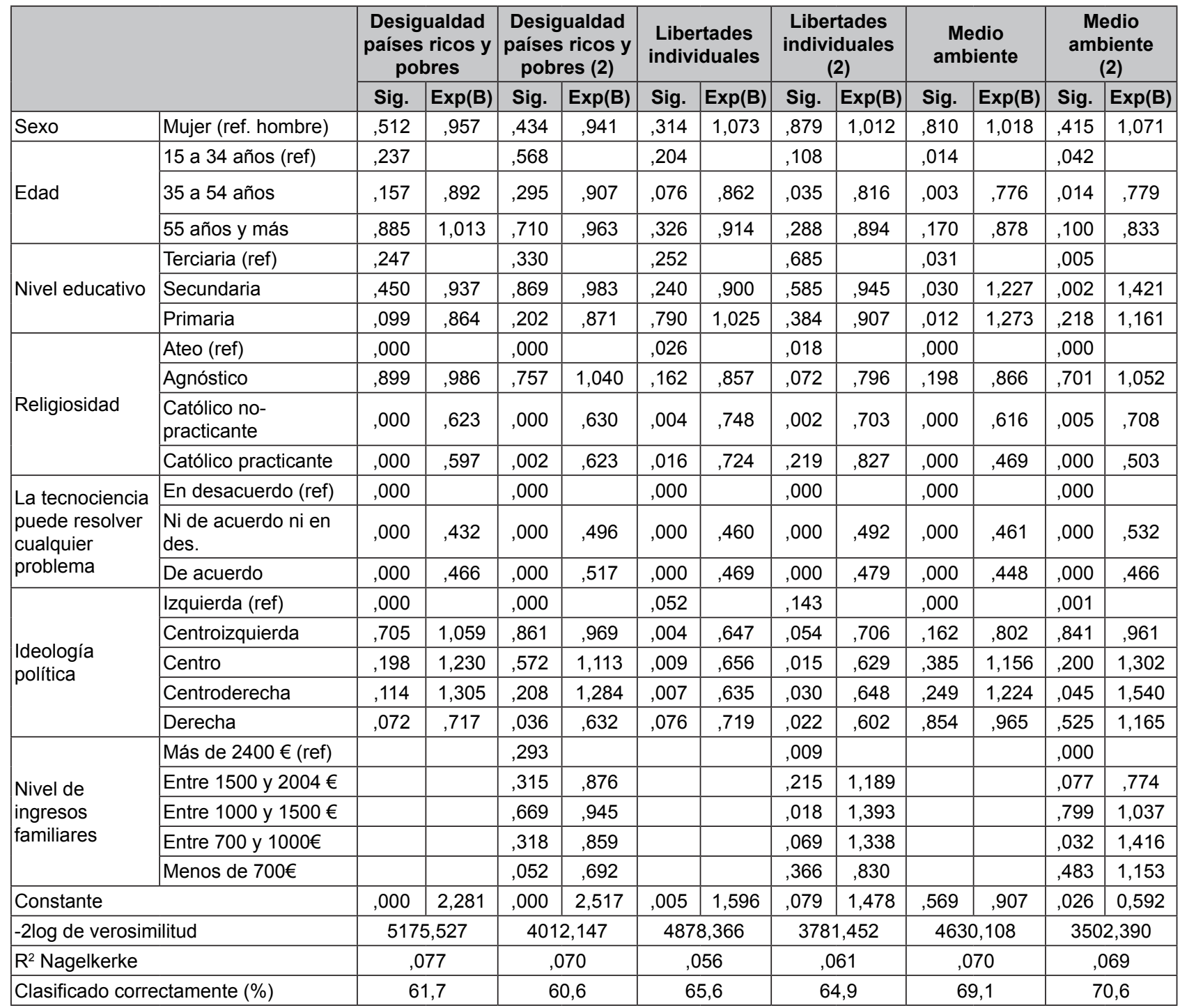

Nota: La categoría de referencia de las variables dependientes es 'Más bien ventajas'. 


\section{Conclusiones}

En nuestro trabajo, constatamos la problematización de ciertos aspectos de la tecnociencia, así como el papel central que ocupa la ambivalencia para la caracterización de sus representaciones sociales. Las sociedades contemporáneas -y la española entre ellas- se han alejado de la valoración meliorativa de la tecnociencia, en un proceso en el que se han desarrollado posiciones ambivalentes a lo largo del último medio siglo (Torres-Albero 2005a; 2005b; 2007; 2009), en consonancia con la cotidianeidad y diversidad de los usos sociales de la tecnociencia. No todas las aplicaciones tecnocientíficas son automáticamente valoradas positivamente por una mayoría de la población, que aplica esquemas cognitivos a la hora de valorar las bondades o desventajas en cada caso. Estos esquemas previos se superponen o, en ocasiones, incluso superan al grado de conocimiento y comprensión tecnocientífica a la hora de explicar dichas posiciones.

Las evidencias que aportamos cuestionan el modelo del déficit cognitivo en las actitudes críticas hacia algunos aspectos de la tecnociencia y ponen de relieve el papel que desempeñan los esquemas cognitivos relacionados con la ideología política, el nivel de religiosidad o la creencia general en la omnipotencia de la tecnociencia. El proceso de secularización de la sociedad en España, por un lado, y el declive de la fe en la omnipotencia de la tecnociencia, por otro, parecen estar asociados con un aumento de las preocupaciones posmaterialistas; y estas, a su vez, con el aumento de la ambivalencia y la problematización de la representación social de la tecnociencia. ¿La religiosidad condiciona la conformación de opiniones en torno a la tecnociencia, más allá de otros factores? Nuestros resultados apuntan a que la religiosidad está conectada a un esquema interpretativo construido en cada individuo que condiciona sus opiniones en torno a la tecnociencia. Afirmamos que la religiosidad, por sí misma, no condiciona la interpretación de los hechos complejos, sino que lo hace la forma en la que esta religiosidad ha sido construida socialmente y a qué sistema de valores se ancla en esa construcción. Así, en culturas como la española, los mayores niveles de religiosidad se manifiestan asociados con actitudes generalmente más positivas de las aplicaciones tecnocientíficas controvertidas -a excepción de la clonación. En cambio, los niveles más bajos de religiosidad se asocian con actitudes más escépticas y críticas respecto a las aplicaciones tecnocientíficas controvertidas, así como a los valores posmaterialistas. Los estudios realizados entre la opinión pública estadounidense muestran cómo la religiosidad tiene un sentido inverso al que detectamos aquí para la sociedad española.

A la hora de explicar su influencia en la conformación de posicionamientos en torno a aspectos controvertidos de la tecnociencia, nuestras conclusiones apuntan a la importancia de comprender cómo la religiosidad se ha construido socialmente en cada cultura, pudiendo estar asociada a sistemas de valores opuestos en diferentes contextos sociales. Esto sugiere que la religiosidad está fuertemente asociada con lógicas prácticas o habitus distintos, en una asociación específica para cada cultura. Así, el efecto de la religiosidad hacia las aplicaciones tecnocientíficas no presentaría una dirección única, contrariamente a lo sugerido en estudios anteriores (Scheufele et al. 2009; Brossard et al. 2009) sino que, al contrario, se muestra en sentidos contrarios en contextos socioculturales distintos. Así, la consideración de la religiosidad como variable nos sirve como identificador del clivaje entre grupos con habitus diferentes. Grupos que han construido lógicas diferentes de interpretar el mundo en cada cultura y en los que la religiosidad aparece como un indicador visible de estas distintas urdimbres de significados.

En las sociedades contemporáneas, la tecnociencia es escasamente cuestionada en su contribución a la mejora de la calidad de vida, las condiciones de salud y el desarrollo económico. Se observa, en cambio, una mayor problematización de su impacto sobre el medio ambiente, las relaciones interpersonales, la libertad y la solidaridad. Las preocupaciones o actitudes críticas no se dirigen hacia la función social de la ciencia ni hacia los científicos como profesión, dado que ambas cuestiones gozan de un elevado prestigio entre la población (Lobera y Torres-Albero 2015). Una proporción significativa de la población recela, sin embargo, de la influencia de intereses privados sobre la tecnociencia a través de su financiación.

La complejidad de las aplicaciones tecnológicas aumenta rápidamente con su especialización, así como la incertidumbre y sus impactos potenciales (Beck 1986). En algunos casos, la comprensión de sus impactos y posibles riesgos se ve reducido a un número limitado de especialistas y no siempre se establece un consenso científico en torno a sus eventuales repercusiones sociales y/o ambientales. En un contexto social en el que la tecnociencia ha perdido su halo de perfectibilidad entre una amplia mayoría de la población, la evaluación que los individuos realizan de las aplicaciones tecnocientíficas complejas está conectada con esquemas o atajos cognitivos anclados en su ideología política, su religiosidad o su creencia en la capacidad general de la tecnociencia. Las diferentes aplicaciones tecnocientíficas presentan características específicas que interactúan con los valores y maneras de entender el mundo de distintos grupos sociales, planteando conflictos específicos en función de esas características en cada sector social con niveles de religiosidad o ideologías políticas diferenciadas.

Ante cuestiones de mayor complejidad, los esquemas cognitivos adquieren una mayor importancia en las actitudes hacia la tecnociencia y disminuye el 
peso del nivel de comprensión o conocimiento científico en la conformación de los posicionamientos. Los individuos se basan, así, en sus valores e ideología para orientarse en sus posiciones en torno a las aplicaciones tecnocientíficas, como esquemas cognitivos de una realidad compleja. Esta consideración de las representaciones sociales de la tecnociencia está alejada del contrato social implícito clásico a favor de la tecnociencia y apunta a la necesidad de que emerja, de manera explícita, un nuevo contrato social acorde con la presencia de diferentes sistemas de valores en las sociedades contemporáneas avanzadas $y$, particularmente, con la cada vez mayor consideración de las preocupaciones posmaterialistas asociadas a la tecnociencia.

\section{NOTAS}

1. La ficha técnica puede ser consultada en: http://www.idi.mineco.gob.es/stfls/MICINN/Prensa/NOTAS_PRENSA/2015/ Dossier_PSC 2015.pdf. El cuestionario completo pueden ser consultado en: http://icono.fecyt.es/informesypublicaciones/Paginas/Percepcion-Social-de-la-Ciencia.aspx

\section{RefERencias Bibliográficas}

Bachelard, G. 1953. La materialisme rationel. París: PUF.

Bauer, M., K. Petkova and P. Boyadjieva. 2000. "Public knowledge of attitudes to science: Alternative measures that may end the 'science war'". Science, Technology \& Human Values 25: 30-51. http://dx.doi. org/10.1177/016224390002500102

Beck, U. 1986. Risikogesellschaft. Auf dem Weg in eine andere Moderne. Fráncfort: Suhrkamp.

Betz, H-G. 1990. "Value Change and Posmaterialist Politics", Comparative Political Studies 23: 239-256. http://dx.doi. org/10.1177/0010414090023002004

Blanco, J. R. y J. M. Iranzo. 2000. "Ambivalencia e incertidumbre en las relaciones entre ciencia y sociedad". Papers 61: 89-112.

Bodmer, W. 1985. The Public Understanding of Science. London: The Royal Society.

Brossard, D. and J. Shanahan. 2003. "Do Citizens Want to Have their Say? Media, Agricultural Biotechnology, and Authoritarian Views of Democratic Processes in Science". Mass Communication and Society 6 (3): 291-312. http://dx.doi.org/10.1207/ S15327825MCS0603_4

Brossard, D. et al. 2009. "Religiosity as a perceptual filter: Examining processes of opinion formation about nanotechnology". Public Understanding of Science 18 (5): 546-558. http://dx.doi.org/10.1177/0963662507087304

Dake, K. 1991. "Orienting dispositions in the perception of risk: an analysis of contemporary worldviews and cultural biases". Journal of Cross-Cultural Psychology 22: 6182. http://dx.doi.org/10.1177/0022022191221006

Douglas, M. and A. Wildavsky. 1982. Risk and Culture: An Essay on the Selection of Technical and Environmental Dangers. Berkeley, CA: University of California Press.

Downs, A. 1957. An Economic Theory of Democracy. Nueva York: Harper \& Row.

Eurobarómetro 2005. Europeans, Science and Technology. Special Eurobarometer 224 / Wave 63.1. Bruselas: Comisión Europea.
Finalmente, consideramos que es conveniente continuar esta línea de investigación con estudios de carácter más cualitativo, que permitieran contrastar los resultados empíricos obtenidos con los discursos y prácticas de los sujetos, así como con datos comparativos sobre las hipótesis que se han probado en este artículo, tanto en nuestro entorno sociocultural más próximo, como de otras sociedades diferentes.

\section{Agradecimientos}

Este texto se integra dentro del proyecto CSO2012-35688 del Plan Nacional de $1+D+\mid$ del Ministerio de Economía y Competitividad (España).

2. Cuando no se incluyen terceras variables ideológicas un mayor nivel de religiosidad entre los individuos españoles está asociado a una mejor valoración de los beneficios de todas las aplicaciones tecnocientíficas testadas, excepto de la clonación.

Fiske, S. T. and P. W. Linville. 1980. "What does the schema concept buy us?" Personality and Social Psychology Bulletin 6: 543-557.

Gabriel, O. W. and J. W. Van Deth. 1995. "Political Interest". Pp. 390-411 in The Impact of Values, edited by J. W. Van Deth y E. Scarbrough. Oxford: Oxford University Press.

Gaskell, G. et al. 2005. "Social values and the governance of science". Science 310: 1908-1909. http://dx.doi. org/10.1126/science. 1119444

(De) Graaf, N. D. and G. Evans. 1996. "Why are the Young more Post-materialist? A Cross-National Analysis of Individual and Contextual Influences on Posmaterial Values". Comparative Political Studies 28: 608-635. http:// dx.doi.org/10.1177/0010414096028004005

Ho, S. S., D. Brossard and D. A. Scheufele. 2008. "Effects of value predispositions, mass media use, and knowledge on public attitudes toward embryonic stem cell research". International Journal of Public Opinion Research 20: 171-192. http://dx.doi.org/10.1093/ ijpor/edn017

Inglehart, R. 1995. "Public Support for Environmental Protection: Objective Problems and Subjective Values in 43 Societies". Political Science and Politics 28: 57-72. http://dx.doi.org/10.1017/S1049096500056080

Inglehart, R. and H. D. Klingemann. 1976. "Party identification, ideological preference and the left-right dimension among western mass publics". Pp. 243-273 in Party Identification and Beyond. Representations of Voting and Party Competition, edited by I. Budge, I. Crewe and D. Farlie. Londres: John Wiley \& Sons.

Johnson, B. B. 1993. "Advancing Understanding of Knowledge's Role in Lay Risk Perception". Risk, Issues in Health and Safety 4:189-212.

Kerschner, C. and M. H. Ehlers. 2016. "A framework of attitudes towards technology in theory and practice". Ecological Economics 126: 139-151.

Kumlin, S. 2001. "Ideology-driven opinion formation in Europe: The case of attitudes towards the third sector 
in Sweden". European Journal of Political Research 39: 487-518

Lobera, J. and C. Torres Albero. 2015. "El prestigio social de las profesiones tecnocientíficas". Pp. 217-240 en Percepción Social de la Ciencia y la Tecnología en España 2014. Madrid: FECYT.

Lyotard, J.-F. 1984. The Postmodern Condition. Minneapolis: University of Minnesota Press.

Martinez, M. D., S. C. Craig and J. G. Kane. 2005. "Pros and Cons: Ambivalence and Public Opinion". Pp. 1-14 in Ambivalence and the Structure of Political Opinion, edited by S. C. Craig and M. D. Martinez. Gordonsville, VA: Palgrave Macmillan.

Moscovici, S. 1984. "The phenomenon of social representations". Pp. 3-69 in Social representations, edited by F. Farr y S. Moscovici. Cambridge: Cambridge University Press.

Priest, S.H. 2001. "Misplaced Faith: Communication Variables as Predictors of Encouragement for Biotechnology Development". Science Communication 23: 97-110. http:// dx.doi.org/10.1177/1075547001023002002

Scheufele, D. A. et al. 2007. "Scientists worry about some risks more than the public". Nature Nanotech 2: 732-734. http://dx.doi.org/10.1038/nnano.2007.392

Scheufele, D. A. et al. 2009. "Religious beliefs and public attitudes toward nanotechnology in Europe and the United States". Nature Nanotech 4: 91-94. http://dx.doi. org/10.1038/nnano.2008.361

Siegrist, M. et al. 2000. "Salient value similarity, social trust, and risk/benefit perception". Risk Analysis 20: 353362. http://dx.doi.org/10.1111/0272-4332.203034
Sniderman, P. M., R. A. Brody and P. E. Tetlock. 1991. Reasoning and Choice: Explorations in Political Psychology. Nueva York: Cambridge University Press. http:// dx.doi.org/10.1017/CBO9780511720468

Sturgis, P. and N. Allum 2004. "Science in Society: Re-evaluating the Deficit Model of Public Attitudes". Public Understanding of Science 13: 55-74. http://dx.doi. org/10.1177/0963662504042690

Torres Albero, C. 2005a. "Representaciones sociales de la ciencia y la tecnología". Revista Española de Investigaciones Sociológicas 111: 9-43. http://dx.doi. org/10.2307/40184698

Torres Albero, C. 2005b. "La ambivalencia ante la ciencia y la tecnología”. Revista Internacional de Sociología 42: 9-38.

Torres Albero, C. 2007. "Estructuras y representaciones sociales de la tecnociencia: el declive de la imagen ilustrada". Pp.185-202 en Percepción Social de la Ciencia y la Tecnología en España 2007. Madrid: FECYT.

Torres Albero, C. 2009. "Las imágenes sociales de la tecnociencia: el caso de España". Redes 30: 149-167.

Van Deth, J. W. and E. Scarbrough. 1995. The impact of values. Vol. 4. Oxford: Oxford University Press.

Wald, K., and Smidt, C. 1993. "Measurement strategies in the study of religion and politics". Pp. 26-49 in Rediscovering the Religious Factor in American Politics, edited by D. Geege \& L. A. Kellstedt. Armonk, NY: M.E. Sharpe

Zaller, J. R. 1992. The Nature and Origins of Mass Opinion. Cambridge: Cambridge University Press.

Ziman, J. 1991. "Public understanding of science". Science, Technology and Human Values 16: 99-105.

CRISTÓBAL TORRES ALBERO es catedrático de universidad en el Departamento de Sociología de la Universidad Autónoma de Madrid. Su campo de trabajo principal es la sociología del conocimiento, de la ciencia y la tecnología, así como el análisis del conjunto de fenómenos sociales asociados al desarrollo de la sociedad de la información y del conocimiento. Entre sus publicaciones más recientes destaca la edición del libro España 2015. Situación social, publicado por el CIS.

JOSEP A. LOBERA es profesor ayudante doctor en el Departamento de Sociología de la Universidad Autónoma de Madrid y docente de Tufts University en su programa en España. Sus campos de trabajo principales son la sociología de la tecnología y el cambio social. Es coordinador científico del informe "Percepción social de la ciencia y la tecnología 2016" editado por la FECYT. 